\title{
CATEGORY AND GROUP RINGS IN HOMOTOPY THEORY
}

\author{
WILLIAM J. RALPH
}

\begin{abstract}
It frequently arises in algebraic topology that a function $\beta: G \rightarrow H$, between two groups, is not a homomorphism. We show that in many standard situations $\beta$ induces a group homomorphism $\bar{\beta}: \mathbf{Z}(G) / \mathscr{A}^{d} \rightarrow H$, where $\mathscr{A}^{d}$ is a power of the augumentation ideal in the group $\operatorname{ring} \mathbf{Z}(G)$. A typical example is $\beta$ : $[X, Y] \rightarrow\left[S^{2} X, S^{2} Y\right]$ where $Y$ is some $H$-group, in which case $d$ can be taken to be $1+$ cat $X$.
\end{abstract}

Introduction. Co- $H$ spaces appear to have a special place in algebraic topology. So many basic maps are homomorphisms only when the space involved is a co- $H$ space. Typical examples of this are the maps $\lambda:[X, Y] \rightarrow\left[S^{n} X, S^{n} Y\right]$ for $n>1$, and $\alpha$ : $[X, Y] \rightarrow \operatorname{Hom}\left(\tilde{h}_{*}(X), \tilde{h}_{*}(Y)\right)$ where $Y$ is an $H$-space and $h_{*}$ is some homology theory. $\lambda$ and $\alpha$ are not homomorphisms in general unless $X$ is a co- $H$ space. Another source of examples is unstable cohomology operations. For example, in ordinary cohomology, $\mu: H^{n}(X, \pi) \rightarrow H^{m}(X, \theta)$ is not a homomorphism in general unless $X$ is a co- $H$ space. One of the major aspects of our theory is to show that the category of $X$ (see [W]) imposes tight restrictions on maps such as $\lambda, \alpha$ and $\mu$.

Recall that a space has category less than or equal to one if and only if it is a co- $H$ space. In a sense we will show that the closer $X$ is to having category 1 , the closer maps like $\lambda, \alpha$ and $\mu$ will be to being homomorphisms.

The algebraic object that comes into play here is a somewhat extended idea of the notion of group ring. As a specific example consider the map $\lambda$ given above and assume that cat $X \leqslant r$ and that $Y$ is an associative $H$-space. A corollary to our theory is that $\lambda$ extends to a homomorphism of abelian groups given by

$$
\bar{\lambda}: \mathbf{Z}([X, Y]) / \mathscr{A}^{r+1} \rightarrow\left[S^{n} X, S^{n} Y\right], \quad n>1,
$$

where $\mathbf{Z}([X, Y])$ is the monoid ring of $[X, Y]$ and $\mathscr{A}$ is the augmentation ideal generated by $\{g-1 \mid g \in[X, Y]\}$. Consider $\bar{\lambda}$ in the case where $r=1$. If $f$, $g \in[X, Y]$ then $(f-1)(g-1) \in \mathscr{A}^{2}$ and hence $f \cdot g-f-g+1 \in \mathscr{A}^{2}$. Applying $\bar{\lambda}$ we have

$$
\begin{aligned}
0 & =\bar{\lambda}(f \cdot g)-\bar{\lambda}(f)-\bar{\lambda}(g)+\bar{\lambda}(1) \\
& =\lambda(f \cdot g)-\lambda(f)-\lambda(g) \text { in }\left[S^{n} X, S^{n} Y\right] .
\end{aligned}
$$

Received by the editors November 25, 1984 and, in revised form, July 19, 1985. Portions of this paper were presented at the meeting of the AMS in Mobile, Alabama, in 1985.

1980 Mathematics Subject Classification. Primary 55Q99, 55Q05.

(C)1987 American Mathematical Society $0002-9947 / 87 \$ 1.00+\$ .25$ per page 
This says precisely that if cat $X \leqslant 1$ then $\lambda$ is a homomorphism, as is easily verified. Hence we see that $\lambda$ being a homomorphism for cat $X \leqslant 1$ is just a special case of a more general situation.

As an example of the kind of thing one can prove using homomorphisms like $\bar{\lambda}$, suppose cat $X \leqslant r, Y$ is an associative $H$-space, $f \in[X, Y]$ and $f^{m}=1$. Using $\bar{\lambda}$, it can be shown that $m^{r} \lambda(f)=0$, and if $m$ is odd and $r>1$ then $m^{r-1} \lambda(f)=0$. Identical results hold for the maps $\alpha$ and $\mu$ given above.

A nice feature of our theory is that the actual computations are conceptually simple and require little insight. The main disadvantage is that the amount of tedium involved is at least an exponential function of the category.

The first section on relation systems places our theory in a general abstract setting. In a sense it can be regarded as the minimum price to be paid in order to obtain a functorial theory, at least that was the original motivation for its development.

$\$ 2$ explores the connections between the groups defined in $\$ 1$ and some of the standard constructions of ordinary homotopy theory. We show for example that the stable versions of our groups are just stable homotopy in many cases. We expect that those relation systems that do not give rise to stable homotopy will prove to be very interesting in the future.

$\$ 3$ shows that there are several different ring structures on our basic groups and gives the rather hard combinatorial proof of Theorem (3.7) which shows how all these ring structures are interconnected. Theorem (3.7) is the key to all the applications of this theory.

The last four sections deal with various applications of the theory. The final application sheds some light on the connection between framed manifolds and the associated elements in the stable homotopy ring of spheres.

1. Relation systems. Throughout this paper all spaces are countable C. W. complexes with basepoints and all maps of spaces are basepoint preserving. Let $\mathscr{T}$ be the category whose objects are pairs $(X, Y)$, where $X$ and $Y$ are any spaces, and whose morphisms $(m, n):(X, Y) \rightarrow(W, Z)$ are pairs of continuous maps $m: W \rightarrow X$ and $n: Y \rightarrow Z$. Let $\mathscr{G}$ be the category of groups and let $\mathscr{S}$ be the category of sets. Let $L: \mathscr{T} \rightarrow \mathscr{S}$ be the functor given by $L(X, Y)=\operatorname{Hom}(X, Y)=\{f \mid f: X \rightarrow Y$ and $f$ continuous $\}$.

(1.1) Definition. A relation system $(\eta, R)$ is a pair consisting of a functor $R$ : $\mathscr{T} \rightarrow \mathscr{G}$ and a natural transformation $\eta: L \rightarrow J \circ R$, where $J$ is the forgetful functor. If in addition $\eta$ always maps the constant map to the identity element of the group then we will call $(\eta, R)$ a reduced relation system.

In the future we will supress $J$ and always regard $\eta: \operatorname{Hom}(X, Y) \rightarrow R(X, Y)$ as a map of sets.

(1.2) Definition. For any relation system $(\eta, R)$ there is a corresponding reduced relation system $(\bar{\eta}, \bar{R})$ obtained by defining $\bar{R}(X, Y)=R(X, Y) /\langle\eta(e)\rangle$, where $\langle\eta(e)\rangle$ is the subgroup generated by the image of the constant map $e$ under $\eta$.

Notations. (i) We will use the symbol $e$ for the constant map or a basepoint, depending on the context. 
(ii) A relation system $(\eta, R)$ will usually be abbreviated by $R$ and the corresponding reduced relation system by $\bar{R}$.

(iii) $F(X)$ will denote the free group on the set $X$ and $\mathbf{Z}(X)$ the free abelian group on the set $X$.

(1.3) Definition. For any relation system $(\eta, R)$ we have the commutative diagram

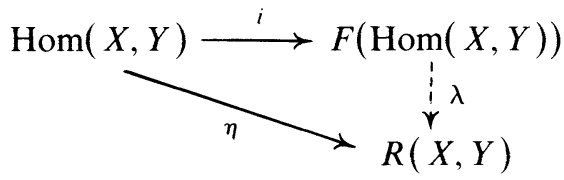

for a unique homomorphism $\lambda$. We define $M(X, Y ; R)$ to be the kernel of $\lambda$

(1.4) EXAMPLES OF RELATION SySTEMS. In each case we will only give the functor $R$, the natural transformation being obvious.

(a) $S$, where $S((X, Y))=\{f \mid f: X \rightarrow F(Y)\}$ under pointwise multiplication;

(b) $T$, where $T((X, Y))=F(\operatorname{Hom}(X, Y)) /\langle M(X, Y ; S) \cup[]$,$\rangle where [, ] denotes$ the commutator subgroup of $F(\operatorname{Hom}(X, Y))$;

(c) $U$, where $U((X, Y))=\{f \mid f: X \rightarrow \mathbf{Z}(Y)\}$ under pointwise multiplication;

(d), (e) and (f) are $\bar{S}, \bar{T}$ and $\bar{U}$, respectively;

(g) $S^{*}$, where $S^{*}((X, Y))=\{f \mid f: X \rightarrow F(Y) /\langle e\rangle\}$;

(h) $T^{*}$, where $T^{*}((X, Y))=F(\operatorname{Hom}(X, Y)) /\left\langle M\left(X, Y ; S^{*}\right) \cup[],\right\rangle$;

(i) $U^{*}$, where $U^{*}((X, Y))=\{f \mid f: X \rightarrow \mathbf{Z}(Y) /\langle e\rangle\}$.

(1.5) DeFinition.

$$
[M(X, Y ; R)]=\left\{\Pi\left[f_{i}\right]^{v_{i}} \in F([X, Y]) \mid \Pi f_{i}^{v_{i}} \in M(X, Y ; R)\right\} .
$$

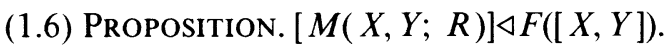

Proof. Immediate from (1.3).

The next idea is the central definition of this paper. The reader should notice that it can be applied in any category whose underlying objects are sets and whose morphisms are (equivalence classes of) functions.

(1.7) Definition. For any relation system $R$ we define a functor $\omega_{R}: \mathscr{T} \rightarrow \mathscr{G}$ by

(1.8) $\omega_{R}(X, Y)=F([X, Y]) /[M(X, Y ; R)]$.

(1.9) Definition. By a map of relation systems $\theta:\left(\eta_{1}, R_{1}\right) \rightarrow\left(\eta_{2}, R_{2}\right)$ we mean a natural transformation $\theta: R_{1} \rightarrow R_{2}$ such that the following commutes:

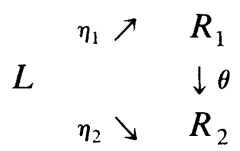

We will denote the category of relation systems by $\mathscr{R}$.

(1.10) Proposition. $\omega$ is a functor from $\mathscr{R}$ to the category of functors from $\mathscr{T}$ to $\mathscr{G}$ and natural transformations between them. 
(1.11) Proposition. If $\theta: R_{1} \rightarrow R_{2}$ is a map of relation systems, then

$$
\omega_{R_{1}}(X, Y) \stackrel{\omega_{\theta}}{\rightarrow} \omega_{R_{2}}(X, Y)
$$

is an epimorphism.

Note, in particular, the following diagram of relation systems (see (1.4)):

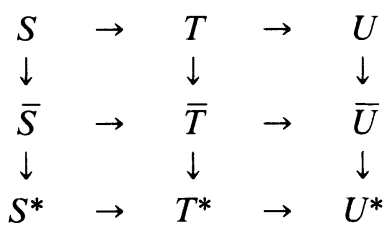

\section{Connections with homotopy.}

(2.1) Proposition. If $X$ is a co-H space, then

(i) the obvious map $[X, Y] \rightarrow \omega_{\bar{S}}(X, Y)$ is an epimorphism;

(ii) $\omega_{\bar{S}}(X, Y)$ is abelian.

Proof. (i) Let $\mu: X \rightarrow X \vee X$ denote the coproduct on $X$. The product $[f] \cdot[g]$ of two classes in $[X, Y]$ is the class of the composite

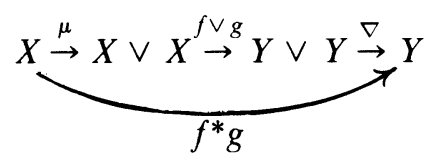

A good exercise for the reader is the proof that

$$
\left(f^{*} e\right)^{-1} \cdot\left(f^{*} g\right) \cdot\left(e^{*} g\right)^{-1} \cdot\left(e^{*} e\right) \in M(X, Y ; \bar{S}) .
$$

Then, since $e \in M(X, Y ; \bar{S})$, we have that $\left[f^{*} g\right]=\left[\left(f^{*} e\right)\right] \cdot\left[\left(e^{*} g\right)\right]$ in $\omega_{\bar{S}}(X, Y)$ and hence $\left[f^{*} g\right]=[f] \cdot[g]$ in $\omega_{\bar{S}}(X, Y)$ since $X$ is co- $H$.

(ii) Follows from the proof of (i) upon noting that

$$
\left(e^{*} g\right)^{-1} \cdot\left(f^{*} g\right) \cdot\left(f^{*} e\right)^{-1} \cdot\left(e^{*} e\right) \in M(X, Y ; \bar{S}) .
$$

(2.2) Proposition. Let $R$ be any of the examples in (1.12). The suspension map, $\omega_{R}(X, Y) \rightarrow \omega_{R}(S X, S Y)$ is a well defined homomorphism.

Proof. We will prove this for $T^{*}$, the proofs for the other systems being similar.

Suppose $\Pi f_{i}^{v_{i}} \in M\left(X, Y ; T^{*}\right)$. It is enough to show that

$$
\prod\left(S f_{i}\right)^{v_{i}} \in M\left(S X, S Y ; T^{*}\right) .
$$

Now $\Pi_{i} f_{i}^{v_{i}}=\Pi_{j} g_{j}^{w_{j}} \cdot \Pi_{k} c_{k}$ in $F(\operatorname{Hom}(X, Y))$, where the $g_{j}$ are generators, the $c_{k}$ are commutators and $\Pi_{j} g_{j}^{w_{j}} \in M\left(X, Y ; S^{*}\right)$. Suspending, we have $\Pi_{i}\left(S f_{i}\right)^{v_{i}=}$ $\Pi_{j}\left(S g_{j}\right)^{w_{j}} \cdot \Pi_{k} S c_{k}$ in $F(\operatorname{hom}(S X, S Y))$, where $c_{k}$ is the commutator obtained by suspending each of the generators in the commutator $c_{k}$. 
It now suffices to show that $\Pi_{j}\left(S g_{k}\right)^{w_{j}} \in M\left(S X, S Y ; S^{*}\right)$. Let $\left(t_{0}, x_{1}\right) \in S^{1} \wedge X$ and let $i_{t_{0}}: X \rightarrow S^{1} \wedge X$ be defined by $i_{t_{0}}(x)=\left(t_{0}, x\right)$. For any $h: X \rightarrow Y$, we have the following commutative diagram of sets and maps:

$$
\begin{array}{ccccc}
X & \stackrel{h}{\rightarrow} & Y & \stackrel{\alpha}{\rightarrow} & F(Y) /\langle e\rangle \\
i_{t_{0}} \downarrow & & i_{t_{0}} \downarrow & & \gamma \downarrow \\
S^{1} \wedge X & \stackrel{S h}{\rightarrow} & S^{1} \wedge Y & \stackrel{\beta}{\rightarrow} & F\left(S^{1} \wedge Y\right) /\langle e\rangle
\end{array}
$$

where $\alpha, \beta$ and $\gamma$ are the obvious maps. Evaluating $\Pi_{j}\left(\beta \circ S g_{j}\right)^{w_{j}}$ at $\left(t_{0}, x_{0}\right)$ we have

$$
\begin{aligned}
\prod_{j}\left(\beta \circ S g_{j}\right)^{w_{j}}\left(t_{0}, x_{0}\right) & =\prod_{j}\left(\beta \circ S g_{j}\left(t_{0}, x_{0}\right)\right)^{w_{j}} \\
& =\prod_{j}\left(\gamma \circ \alpha \circ g_{j}\left(x_{0}\right)\right)^{w_{j}} \quad(\text { by }(2.3)) \\
& =\gamma\left(\prod_{j}\left(\alpha \circ g_{j}\left(x_{0}\right)\right)^{w_{j}}\right) \\
& =\gamma(1) \quad \text { since } \prod_{j} g_{j}^{w_{j}} \in M\left(X, Y ; S^{*}\right) \\
& =1 .
\end{aligned}
$$

The relations we have introduced are defined at the point set level in a very rigid way. They are not, on the surface, compatible with the usual multiplications in homotopy theory which are multiplications only up to homotopy. We get around this by using the following theorem due to Milnor.

THEOREM (SEE [St]). If $Y$ has the homotopy type of a connected countable C.W. complex, then there is a topological group $G(X)$ of the same homotopy type of $\Omega X$.

The following theorem is the central connection between our theory and homotopy theory.

(2.4) THEOREM. Let $Y$ have the homotopy type of a connected countable C.W. complex. Then the obvious map $\omega_{T^{*}}(X, Y) \rightarrow\left[S^{2} X, S^{2} Y\right]$ is a well-defined homomorphism.

Proof. Since $\left[S^{2} X, S^{2} Y\right]$ is abelian it is enough to check that if

$$
\prod f_{i}^{v_{i}} \in M\left(X, Y ; S^{*}\right)
$$

then $\Pi\left(S^{2} f_{i}\right)^{v_{i}=1}$ in $\left[S^{2} X, S^{2} Y\right]$. From the proof of Proposition (2.2) we know that $\Pi\left(S f_{i}\right)^{\prime \prime}, \in M\left(S X, S Y ; S^{*}\right)$. Let $\theta: \Omega^{1} S^{2} Y \rightarrow G\left(S^{2} Y\right)$ be the homotopy equivalence given in Milnor's theorem above. Consider the following commutative diagram of sets

$$
\begin{aligned}
& S X \quad \rightarrow \quad S Y \quad \stackrel{j}{\rightarrow} \quad \Omega^{1} S^{2} Y \quad \stackrel{\theta}{\rightarrow} \quad G\left(S^{2} Y\right) \\
& k \downarrow \quad \uparrow \rho \\
& \frac{F(S Y)}{\langle e\rangle} \quad \stackrel{j}{\rightarrow} \frac{F\left(\Omega^{1} S^{2} Y\right)}{\langle e\rangle} \quad \stackrel{\bar{\theta}}{\rightarrow} \frac{F\left(G\left(S^{2} Y\right)\right)}{\langle e\rangle}
\end{aligned}
$$


where $k$ and $j$ are the inclusions, $\bar{j}$ and $\bar{\theta}$ are the induced maps and $\rho$ is the obvious homomorphism. Now for any $x \in X$ we have

$$
\begin{gathered}
\prod_{i}\left(\left(\theta \circ j \circ S f_{i}\right)(x)^{v_{i}}\right. \\
=\prod_{i}\left(\left(\rho \circ \bar{\theta} \circ \bar{j} \circ k \circ S f_{i}\right)(x)\right)^{v_{i}} \\
=\rho \circ \bar{\theta} \circ \bar{j}\left(\prod_{i}\left(\left(k \circ S f_{i}\right)(x)\right)^{v_{i}}\right) \quad(\text { since } \rho \circ \bar{\theta} \circ \bar{j} \text { is a homomorphism }) \\
=\rho \circ \bar{\theta} \circ \bar{j}(1) \quad \text { since } \prod_{\left(S f_{i}\right)^{v_{i}} \in M\left(X, Y ; S^{*}\right)} \\
=1 . \quad \therefore \prod_{i}\left[S^{2} f_{i}\right]^{v_{i}}=1 . \quad \square
\end{gathered}
$$

(2.5) Proposition. Let $X$ be any space. Suppose that $Y$ is a space such that there is an $H$-space equivalence between $\Omega S Y$ and some topological group. Let $G=[S X, S Y]$. Then the obvious map $\omega_{T^{*}}(X, Y) \rightarrow G /[G, G]$ is a well-defined homomorphism.

Proof. This is a straightforward modification of the proof of (2.4).

(2.6) Definition. Let $R$ be any of the relation systems in (1.12). By (2.2) we may define $\omega_{R}^{s}(X, Y)=\lim _{n} \omega_{R}\left(S^{n} X, S^{n} Y\right)$.

(2.7) TheOREM. Let $R$ be one of $\bar{S}, S^{*}, \bar{T}$ or $T^{*}$. Let $Y$ be a $C$. W. complex and let $\{X, Y\}$ denote the group of stable homotopy classes of maps from $X$ to $Y$. Then $\omega_{R}^{s}(X, Y) \simeq\{X, Y\}$.

Proof. By (2.1) and (1.11) there is an epimorphism $\{X, Y\} \rightarrow \omega_{R}^{s}(X, Y)$ and the following commutative diagram:

$$
\begin{array}{ccccc}
\{X, Y\} & \rightarrow & \omega_{S}^{s}(X, Y) & \rightarrow & \omega_{S^{*}}^{s}(X, Y) \\
& \downarrow & & \downarrow \\
& \omega_{T}^{s}(X, Y) & \rightarrow & \omega_{T^{*}}^{s}(X, Y)
\end{array}
$$

Hence it is enough to show that $\{X, Y\} \rightarrow \omega_{T^{*}}^{s}(X, Y)$ has no kernel. But this is immediate from (2.4) which supplies the inverse.

(2.8) THEOREM. The kernel of $\pi_{n}(X) \rightarrow \bar{\omega}\left(S^{n}, X\right)$ contains all Whitehead products.

Proof. We will use the notation of $(22)$ of $[\mathbf{R}]$. It is easy to check that $\{f, e\} \cdot\{f, g\}^{-1} \cdot\{e, g\} \cdot\{e, e\}^{-1} \in M\left(S^{n}, Y ; \bar{S}\right)$. By standard properties of Whitehead products $[f, 0]=0=[0, g]$. The result now follows.

(2.9) ThEOREM. (1) Let $G$ be a topological group. Then:

(a) $\omega_{S^{*}}(X, G) \rightarrow[X, G]$ is a well-defined epimorphism.

(b) If $X$ is a co- $H$ space, then all the maps in the following diagram are isomorphisms:

$$
\begin{aligned}
& \omega_{\bar{S}}(X, G) \quad \rightarrow \quad \omega_{S^{*}}(X, G) \\
& \downarrow \\
& \omega_{\bar{T}}(X, G) \quad \rightarrow \quad \omega_{T^{*}}(X, G) \quad \rightarrow \quad[X, G]
\end{aligned}
$$


(c) If $[X, G]$ is abelian, then $\omega_{T^{*}}(X, G) \rightarrow[X, G]$ is a well-defined homomorphism.

(2) Let $G$ be an abelian topological group (i.e., a generalized Eilenberg-Mac Lane space if $G$ is connected, see [St]). Then:

(a) $\omega_{U^{*}}(X, G) \rightarrow[X, G]$ is a well-defined epimorphism.

(b) If $X$ is a co- $H$ space, then all the maps in the following diagram are isomorphisms:

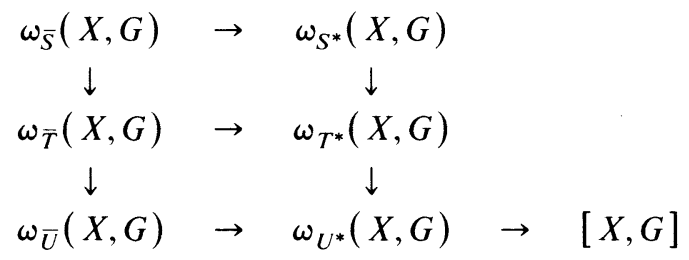

The proofs of these assertions are straightforward and left to the reader. In part (2) it is unknown whether abelian can be replaced by homotopy abelian.

Next we give a factorization of the Hurewicz map that shows that several of our constructions are not too pathological in the sense that they are bounded above by homotopy and below by homology. Our proof requires the main result of $[\mathbf{R}]$, namely Theorem (18). The following definition and proposition are introduced so as to make a bridge to [R].

(2.10) Definition. Let $f_{i}: X \rightarrow Y, i \in I$. We set $I(x, y)=\left\{i \mid f_{i}(x)=y\right\}$.

(2.11) Proposition. $\Pi f_{i}^{v_{i}} \in M(X, Y ; U) \Leftrightarrow \sum_{I(x, y)} v_{i}=0$ for all $(x, y) \in X \times Y$.

Proof. Straightforward.

(2.12) TheOREM. The Hurewicz map $\rho: \pi_{n}(X) \rightarrow H_{n}(X)$ factors as follows:

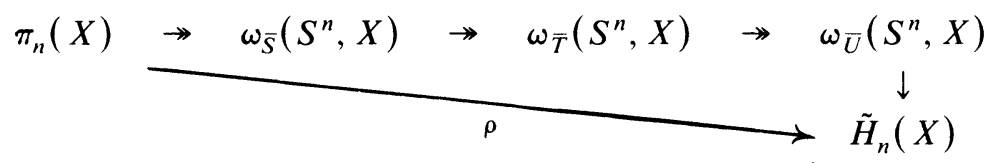

Proof. Using (2.11) this follows from (11), (18) and (28) of [R].

REMARK. It is easy to see that the graded group $\omega_{U}^{S}\left(S^{*} ; S^{\circ}\right)$ is a ring under composition and that there is a ring homomorphism $\pi_{*}{ }^{S}\left(S^{\circ}\right) \rightarrow \omega_{U}^{S}\left(S^{*} ; S^{\circ}\right)$ which is an epimorphism by (1.11), (1.12) and (2.1). We consider the fundamental problem in this theory to be the investigation of the kernel of this map. At this point all that is known is that there is an isomorphism in degree zero, which follows from (2.12).

3. Multirings. In this section we will introduce a family of ring structures on $\omega_{T}(X, Y)$ and show that they are all tightly interconnected.

(3.1) Definition. Let $P(X, Y) \subset \mathbf{Z}([X, Y])$ be the subgroup of sums $\sum_{i} v_{i}\left[f_{i}\right]$ such that there exists $\sum_{j} w_{j}\left[g_{j}\right]$ with $\sum_{i} v_{i}\left[f_{i}\right]=\sum_{j} w_{j}\left[g_{j}\right]$ and $\prod_{j} g_{j}^{w_{j}} \in M(X, Y ; S)$.

(3.2) Proposition. $\omega_{T}(X, Y) \simeq \mathbf{Z}([X, Y]) / P(X, Y)$.

Proof. Trivial. 
(3.3) Proposition. Any map $\mu: Y \times Y \rightarrow Y$ induces a binary operation on $[X, Y]$ and hence a ring structure on $\mathbf{Z}([X, Y])$ (not necessarily associative or having identity). For any such $\mu, P(X, Y)$ is a two-sided ideal in $\mathbf{Z}([X, Y])$.

Proof. Let $\Pi g_{j}^{w_{i}} \in M(X, Y ; S)$ and suppose $f: X \rightarrow Y$. Denote by $f \cdot g_{j}$ the following composite:

$$
X \stackrel{\Delta}{\rightarrow} X \times \underset{f \times g_{j}}{\rightarrow} Y \times Y \stackrel{\mu}{\rightarrow} Y .
$$

We claim $\Pi\left(f \cdot g_{j}\right)^{w_{i}} \in M(X, Y ; S)$ and similarly for $\Pi\left(g_{j} \cdot f\right)^{w_{j}}$.

Let $i: Y \rightarrow F(Y)$ be the inclusion. Fix $x \in X$ and suppose $\Delta(x)=\left(x_{1}, x_{2}\right)$. Define a function $\gamma: X \rightarrow Y$ by $\gamma(y)=\mu\left(f\left(x_{1}\right), y\right)$. (Note that $\gamma$ is not necessarily basepoint preserving. It is for this reason that we do not obtain ideals for $\bar{S}$ and $S^{*}$ without further restrictions on $\mu$ ). Let $\bar{\gamma}: F(Y) \rightarrow F(Y)$ be the induced map.

We have

$$
\begin{aligned}
\prod_{j}\left(\left(i \circ\left(f \cdot g_{j}\right)\right)(x)\right)^{w_{j}} & =\prod_{j}\left(\left(i \circ \mu \circ\left(f \times g_{j}\right) \circ \Delta\right)(x)\right)^{w_{j}} \\
& =\prod_{j}\left((i \circ \mu)\left(f\left(x_{1}\right), g_{j}\left(x_{2}\right)\right)\right)^{w_{j}} \\
& =\bar{\gamma}\left(\prod_{j}\left(\left(i \circ g_{j}\right)\left(x_{2}\right)\right)^{w_{j}}\right) \\
& =\bar{\gamma}(1) \quad \text { since } \prod g_{j}^{w_{j}} \in M(X, Y ; S) \\
& =1 .
\end{aligned}
$$

Jargon. We will refer to $\mathbf{Z}([X, Y])$ and $\omega_{T}(X, Y)$, with all the ring structures induced by maps $Y \times Y \rightarrow Y$, as multirings. A subgroup of a multiring will be called a multiideal if it is an ideal with respect to each ring structure. An element of the multiideal $P(X, Y)$ will be called a "transitopy" relation and we will refer to $\omega_{T}(X, Y)$ as the "transitopy multiring".

(3.4) Definition. By the $n$th augmentation multiideal, $\mathscr{A}^{(n)}(X, Y)$, we will mean the multiideal of $\mathbf{Z}([X, Y])$ generated by all possible products of the form

$$
\left(f_{1}-e\right) o_{1}\left(f_{2}-e\right) o_{2} \cdots o_{n-1}\left(f_{n}-e\right)
$$

under any allowable choice of bracketing, where the $o_{i}$ are any $n-1$ of the products on $\mathbf{Z}([X, Y]), f_{i}: X \rightarrow Y$ and $e$ is the constant map.

(3.5) e.g. An allowable choice of bracketing of $\left(f_{1}-e\right) o_{1}\left(f_{2}-e\right) o_{2}\left(f_{3}-e\right)$ would be $\left[\left(f_{1}-e\right) o_{1}\left(f_{2}-e\right)\right] o_{2}\left(f_{3}-e\right)$. Expanding, this becomes

$$
\begin{aligned}
{\left[f_{1} o_{1} f_{2}\right.} & \left.-e o_{1} f_{2}-f_{1} o_{1} e+e o_{1} e\right] o_{2}\left(f_{3}-e\right) \\
= & \left(f_{1} o_{1} f_{2}\right) o_{2} f_{3}-\left(e o_{1} f_{2}\right) o_{2} f_{3}-\left(f_{1} o_{1} e\right) o_{2} f_{3} \\
& +\left(e o_{1} e\right) o_{2} f_{3}-\left(f_{1} o_{1} f_{2}\right) o_{2} e-\left(e o_{1} f_{2}\right) o_{2} e \\
& -\left(f_{1} o_{1} e\right) o_{2} e+\left(e o_{1} e\right) o_{2} e \in \mathscr{A}^{(3)}(X, Y) .
\end{aligned}
$$

In our applications, the power of the augmentation multiideal will be determined by the category of $X$. We give a definition of category that is convenient for our use (see the discussion on p. 456 of $[\mathbf{W}]$ ). 
(3.6) Definition. The category of $X$ is the smallest $c$ for which there is a covering of $X$ by closed sets $A_{1}, \ldots, A_{c+1}$ with the property that there are basepoint preserving homotopies $H_{t}^{i}: X \rightarrow X$ with $H_{0}^{i}=\mathrm{id}_{X}$ and $H_{1}^{i}\left(A_{i}\right)=e$.

The following theorem is our central result. Together with (2.4) it will form the basis for all subsequent applications.

(3.7) TheOREM. If $X$ has category $c$, then $\mathscr{A}^{(c+1)}(X, Y) \subset P(X, Y)$.

Proof. Later.

All the hard work in the proof of (3.7) is contained in the rather complicated combinatorial proof of Lemma (3.12) below. We require some preliminary notations before stating the lemma.

(3.8) Definition. In our definition of $M(X, Y ; T), \operatorname{Hom}(X, Y)$ was the set of all basepoint preserving maps from the space $X$ to the space $Y$. If we now take $X$ and $Y$ to be any sets (without basepoint) and $\operatorname{Hom}(X, Y)$ to be the set of all functions from $X$ to $Y$, we can make the appropriate changes in our earlier definitions (in particular (1.3)) and obtain $M s(X, Y ; T)$, the analogue of $M(X, Y ; T)$.

(3.9) Definition. $\mathscr{J}_{k}=\left\{\left(a_{0}, \ldots, a_{k}\right) \mid a_{i}=0\right.$ or 1$\}$.

(3.10) Definition. Given $\left(a_{0}, \ldots, a_{k}\right) \in \mathscr{J}_{\bar{k}}$ we define $\left[a_{0}, \ldots, a_{k}\right]:\{0, \ldots, k\} \rightarrow$ $\mathscr{J}_{k}$ by

$$
\left[a_{0}, \ldots, a_{k}\right](i)=\left(a_{0}, \ldots, \underset{i}{o}, \ldots, a_{k}\right) .
$$

\section{(3.11) DEFINITION.}

$$
\sigma\left(a_{0}, \ldots, a_{k}\right)=(-1)^{\left(\# \text { of } a_{i}\right. \text { that are zero) }} .
$$

(3.12) LEMMA. $\prod_{\mathscr{J}_{k}}\left[a_{0}, \ldots, a_{k}\right]^{\sigma\left(a_{0}, \ldots, a_{k}\right)} \in M s\left(\{0, \ldots, k\}, \mathscr{J}_{k} ; T\right)$

(Note that this product occurs in a nonabelian group and hence we should specify an ordering of $\mathscr{J}_{k}$ in which the product is to be taken. From the definition of $T$ we see that if the lemma is true for one ordering it is true for all, so there is no ambiguity.)

Proof. By induction on $k$. It is trivial to check the result for $k=0$; however, it is more enlightening to see the proof for $k=1$. From now to the end of the lemma it will be convenient to regard $\left[a_{0}, \ldots, a_{k}\right]^{\sigma\left(a_{0} \ldots, a_{k}\right)}$ as an element of $F\left(\mathscr{J}_{k}\right)^{k+1}$, the cartesian product of $k+1$ copies of the free group on $\mathscr{J}_{k}$.

The case $k=1$ : We have

$$
\begin{aligned}
& {[0,0]^{\sigma(0,0)}=((0,0),(0,0)),} \\
& {[0,1]^{\sigma(0,1)}=\left((0,1)^{-1},(0,0)^{-1}\right),} \\
& {[1,0]^{\sigma(1,0)}=\left((0,0)^{-1},(1,0)^{-1}\right),} \\
& {[1,1]^{\sigma(1,1)}=((0,1),(1,0)) .}
\end{aligned}
$$

To show that the product of these elements is in $M s\left(\{0,1\}, \mathscr{J}_{1} ; T\right)$ we must find a rearrangement such that the product in each coordinate is $1 \in F\left(\mathscr{J}_{1}\right)$. We have

$$
\begin{aligned}
((0,0),(0,0)) \cdot\left((0,1)^{-1},(0,0)^{-1}\right) \cdot((0,1),(1,0)) \cdot\left((0,0)^{-1},(1,0)^{-1}\right) & =1 \\
& \text { in } F\left(\mathscr{J}_{1}\right) \times F\left(\mathscr{J}_{1}\right) .
\end{aligned}
$$


(The reader should do the cases $k=2$ and 3 to really appreciate the difficulties this induction will surmount.)

Now assume the lemma proven for $k=n$. For $\left(a_{1}, \ldots, a_{n+1}\right) \in \mathscr{J}_{n}$ we define $B\left(a_{1}, \ldots, a_{n+1}\right) \in F\left(\mathscr{J}_{n+1}\right)^{n+2}$ as follows:

$$
B\left(a_{1}, \ldots, a_{n+1}\right)=\left\{\begin{array}{r}
{\left[1, a_{1}, \ldots, a_{n+1}\right]^{\sigma\left(1, a_{1}, \ldots, a_{n+1}\right)}\left[0, a_{1}, \ldots, a_{n+1}\right]^{\sigma\left(0, a_{1}, \ldots, a_{n+1}\right)}} \\
\text { if } \sigma\left(1, a_{1}, \ldots, a_{n+1}\right)=1, \\
{\left[0, a_{1}, \ldots, a_{n+1}\right]^{\sigma\left(0, a_{1}, \ldots, a_{n+1}\right)}\left[\begin{array}{r}
\left.1, a_{1}, \ldots, a_{n+1}\right]^{\sigma\left(1, a_{1}, \ldots, a_{n+1}\right)} \\
\text { if } \sigma\left(1, a_{1}, \ldots, a_{n+1}\right)=-1 .
\end{array}\right.}
\end{array}\right.
$$

Note that to prove the lemma for $k=n+1$ it suffices to find an arrangement of all the $B\left(a_{1}, \ldots, a_{n+1}\right)$, whose product is 1 in $F\left(\mathscr{J}_{n+1}\right)^{n+2}$.

The first coordinate of $B\left(a_{1}, \ldots, a_{n+1}\right)$ is either

$$
\left(0, a_{1}, \ldots, a_{n+1}\right)^{\sigma\left(1, a_{1}, \ldots, a_{n+1}\right)}\left(0, a_{1}, \ldots, a_{n+1}\right)^{\sigma\left(0, a_{1}, \ldots, a_{n+1}\right)}
$$

or these terms in the reverse order. In either case $\sigma\left(1, a_{1}, \ldots, a_{n+1}\right)+$ $\sigma\left(0, a_{1}, \ldots, a_{n+1}\right)=0$ so their product is $1 \in F\left(\mathscr{J}_{n+1}\right)$. Therefore we may ignore the first coordinate from now on.

By the induction hypothesis, there is an ordering of all the elements of $\mathscr{J}_{n}$ such that

$$
\prod_{\mathscr{J} n}\left[a_{1}, \ldots, a_{n+1}\right]^{\sigma\left(a_{1}, \ldots, a_{n+1}\right)}=1 \text { in } F\left(\mathscr{J}_{n}\right)^{n+1} .
$$

The symbol $\Pi_{\mathscr{J}_{n}}$ will be in reference to this particular ordering from now on.

We claim $\prod_{\mathscr{Z}_{n}} B\left(a_{1}, \ldots, a_{n+1}\right)=1$ in $\left(F\left(\mathscr{J}_{n+1}\right)\right)^{n+2}$. This has already been shown to be true in the first coordinate. Let $j=2,3, \ldots$, or $n+1$ be any other coordinate. This $j$ will be fixed from now on.

Each term $\left[a_{1}, \ldots, a_{n+1}\right]^{\sigma\left(a_{1}, \ldots, a_{n+1}\right)}$ will have coordinate $x_{i}$, say, in position $j-1$ and hence we can write the coordinate of $\prod_{\mathscr{Z}_{n}}\left[a_{1}, \ldots, a_{n+1}\right]^{\sigma\left(a_{1}, \ldots, a_{n+1}\right)}$ in position $j-1$ as $x_{1} \cdot x_{2} \cdots x_{2^{n+1}} \in F\left(\mathscr{J}_{n}\right)$. Similarly each term $B\left(a_{1}, \ldots, a_{n+1}\right)$ will have coordinate $y_{i}$, say, in position $j$ and hence we can write the coordinate of $\Pi_{\mathscr{J}_{n}} B\left(a_{1}, \ldots, a_{n+1}\right)$ in position $j$ as $y_{1} \cdot y_{2} \cdots y_{2^{n+1}} \in F\left(\mathscr{J}_{n+1}\right)$. Our proof will be finished if we can show that $y_{1} \cdot y_{2} \cdots y_{2^{n+1}}=1$ in $F\left(\mathscr{J}_{n+1}\right)$.

Each of the elements $x_{i}$ is either a generator or the inverse of a generator. It is easily checked that a generator and its inverse occur exactly once in the sequence $x_{1}, \ldots, x_{2^{n+1}}$. Since we are working in a free group, this implies, that for $x_{1}, \ldots, x_{2^{n+1}}$ to be 1 , we must have generators and their inverses cancelling in pairs, where each pair is distinct from every other pair. A little thought then shows that to prove $y_{1} \cdots y_{2^{n+1}}=1$ it suffices to show that if $x_{a} \cdot x_{b}=1$ then $y_{a} \cdot y_{b}=1$.

Now fix $\left(c_{1}, \ldots, c_{n}\right) \in \mathscr{J}_{n-1}$. Let $x_{a}$ be the coordinate in position $j-1$ of the term

$$
\left[c_{1}, \ldots, c_{j-2}, 0, c_{j-1}, \ldots, c_{n}\right]^{\left(c_{1}, \ldots, c_{j-2}, 0, c_{j-1}, \ldots, c_{n}\right)} .
$$

The (unique) term that has $x_{b}$, the inverse of $x_{a}$, as the coordinate in position $j-1$ is

$$
\left[c_{1}, \ldots, c_{j-2}, 1, c_{j-1}, \ldots, c_{n}\right]^{\sigma\left(c_{1}, \ldots, c_{j-2}, 1, c_{j-1}, \ldots, c_{n}\right)}
$$


The corresponding terms $y_{a}$ and $y_{b}$ will be the $j$ th coordinates of $B\left(c_{1}, \ldots, c_{j-2}, 0, c_{j-1}, \ldots, c_{n}\right)$ and $B\left(c_{1}, \ldots, c_{j-2}, 1, c_{j-1}, \ldots, c_{n}\right)$, respectively. All we have left is to show is that $y_{a} \cdot y_{b}=1$. Suppose $\sigma\left(1, c_{1}, \ldots, c_{j-2}, 0, c_{j-1}, \ldots, c_{n}\right)$ $=1$, the other case being similar. Then

$$
\begin{aligned}
y_{a}= & \left(1, c_{1}, \ldots, c_{j-2}, 0, c_{j-1}, \ldots, c_{n}\right)^{\sigma\left(1, c_{1}, \ldots, c_{j-2}, 0, c_{j-1}, \ldots, c_{n}\right)} \\
& \cdot\left(0, c_{1}, \ldots, c_{j-2}, 0, c_{j-1}, \ldots, c_{n}\right)^{\sigma\left(0, c_{1}, \ldots, c_{j-2}, 0, c_{j-1}, \ldots, c_{n}\right)}
\end{aligned}
$$

and

$$
\begin{aligned}
y_{b}= & \left(0, c_{1}, \ldots, c_{j-2}, 0, c_{j-1}, \ldots, c_{n}\right)^{\sigma\left(0, c_{1}, \ldots, c_{j-2}, 1, c_{j-1}, \ldots, c_{n}\right)} \\
& \cdot\left(1, c_{1}, \ldots, c_{j-2}, 0, c_{j-1}, \ldots, c_{n}\right)^{\sigma\left(1, c_{1}, \ldots, c_{j-2}, 1, c_{j-1}, \ldots, c_{n}\right)} .
\end{aligned}
$$

Clearly $y_{a} \cdot y_{b}=1$ as required.

PROOF OF (3.7). Fix an element

$$
\left(f_{0}-e\right) o_{1} \cdots o_{c}\left(f_{c}-e\right)
$$

with some choice of bracketing (see (3.4) and (3.5)). Let $A_{i}, i=0, \ldots, c$, be the covering given in (3.6). Choose $g_{i} \sim f_{i}$ with $\left.g_{i}\right|_{A_{i}}=e$.

If $h, h_{i}: X \rightarrow Y$, where $i=0,1, \ldots, c$, we will say $h=\left(h_{0}, \ldots, h_{c}\right)$ if $\left.h\right|_{A_{i}}=\left.h_{i}\right|_{A_{i}}$. $h^{0}$ will denote the constant map $e$. $\prod_{i=0}^{c} g_{i}^{a_{i}}$, where $\left(a_{0}, \ldots, a_{c}\right) \in \mathscr{J}_{c}$, will always be taken with respect to the bracketing and operations of (3.13).

Notice first that for any $\left(a_{0}, \ldots, a_{c}\right) \in \mathscr{J}_{c}$ we have

$$
\prod_{i=0}^{c} g_{i}^{a_{i}}=\left(\prod_{\substack{i=0 \\ a_{0}=0}}^{c} g_{i}^{a_{i}}, \ldots, \prod_{\substack{i=0 \\ a_{c}=0}}^{c} g_{i}^{a_{i}}\right)
$$

since $\left.g_{i}\right|_{A_{i}}=e$. Hence we can write (3.13) as

$$
\sum_{\left(a_{0}, \ldots, a_{c}\right) \in \mathscr{F}_{c}} \sigma\left(a_{0}, \ldots, a_{c}\right)\left(\prod_{\substack{i=0 \\ a_{0}=0}}^{c} g_{i}^{a_{i}}, \ldots, \prod_{\substack{i=0 \\ a_{c}=0}}^{c} g_{i}^{a_{i}}\right) .
$$

It follows that we want to find an ordering of $\mathscr{J}_{c}$ so that:

$$
\prod_{\mathscr{g}_{c}}\left(\prod_{\substack{i=0 \\ a_{i}=0}}^{c} g_{i}^{a_{i}}, \ldots, \prod_{\substack{i=0 \\ a_{c}=0}}^{c} g_{i}^{a_{i}}\right)^{\sigma\left(a_{0} \ldots \ldots a_{c}\right)} \in M(X, Y ; S) .
$$

From now on $\Pi_{\mathcal{J}}$ will refer to the ordering given in Lemma (3.12). We claim that this ordering yields (3.14).

Fix $x \in X . x \in A_{n}$ for some $n$ and this $n$ will be fixed from now on. Define a function from $\mathscr{J}_{c}$ to $Y$ by $\left(a_{0}, \ldots, a_{c}\right) \rightarrow\left(\prod_{i=0}^{c} g_{i}^{a_{i}}\right)(x)$. This extends to the homomorphism $\lambda: F\left(\mathscr{J}_{c}\right) \rightarrow F(Y)$. Since $x \in A_{n}$, to show (3.14) it is enough to show

$$
\prod_{\mathscr{L}_{c}}\left[\left(\prod_{\substack{i=0 \\ a_{n}=0}}^{c} g_{i}^{a_{i}}\right)(x)\right]^{\sigma\left(a_{0} \ldots \ldots a_{c}\right)}=1 \in F(Y) .
$$


We have $\Pi_{\mathscr{J}_{c}}\left[a_{0}, \ldots, a_{c}\right]^{\sigma\left(a_{0}, \ldots, a_{c}\right)}=1$ in $F\left(\mathscr{J}_{c}\right)^{c+1}$. Taking the $n$th coordinate of this we obtain

$$
\prod_{\mathscr{J}_{c}}\left(a_{0}, \ldots, 0_{n}, \ldots, a_{c}\right)^{\sigma\left(a_{0}, \ldots, a_{c}\right)}=1
$$

in $F\left(\mathscr{J}_{c}\right)$. Applying $\lambda$ to (3.16) we obtain (3.15).

4. Applications. We will deal with several situations simultaneously by referring to several different homomorphisms by the symbol. Theorem (4.14), which is given in terms of $\beta$, will then cover all these situations.

First we have the homomorphisms

$$
\omega_{T}(X, Y) \rightarrow\left[S^{1} \wedge X, S^{1} \wedge Y\right] /[,] \text { and } \omega_{T}(X, Y) \rightarrow\left[S^{2} \wedge X, S^{2} \wedge Y\right]
$$

given by (2.4) and (2.5). A connection with homology and cohomology theories is given by

(4.2) Proposition. Let $h_{*}$ and $h^{*}$ be any reduced homology and cohomology theories, respectively. Then the obvious maps

$$
\begin{aligned}
& \quad \omega_{T}(X, Y) \rightarrow \operatorname{Hom}\left(\tilde{h}_{*}(X), \tilde{h}_{*}(Y)\right) \text { and } \omega_{T}(X, Y) \rightarrow \operatorname{Hom}\left(\tilde{h}^{*}(X), \tilde{h}^{*}(Y)\right) \\
& \text { are homomorphisms of abelian groups. }
\end{aligned}
$$

Proof. This is straightforward from (2.5) and standard properties of homology and cohomology theories. See 7.38 of [Sw] for example.

The final situation we have in mind is to use the homomorphism $\omega_{T}(X, G) \rightarrow$ $[X, G]$ given by $(2.9 \mathrm{c})$. This has applications to unstable cohomology operations. For example, in ordinary cohomology, any natural transformation $\lambda: \tilde{H}^{n}(X, \pi) \rightarrow$ $\tilde{H}^{m}(X, \rho)$ is induced by a map $\mathscr{K}(\pi, n) \rightarrow \mathscr{K}(\rho, m)$. Since $\mathscr{K}(\rho, m)$ can be taken to be a topological group we can apply (2.9c) to obtain the following composition of homomorphisms of abelian groups:

$$
\omega_{T}(X, \mathscr{K}(\pi, n)) \rightarrow \omega_{T}(X, \mathscr{K}(\rho, m)) \rightarrow \tilde{H}^{m}(X, \rho) .
$$

(4.4) gives a great deal of information about how close $\lambda$ is to being a homomorphism.

A simplified version of (4.4) can be written in a more standard way as follows. Write $\mathbf{Z}\left(\tilde{H}^{n}(X, \pi)\right)$ for the grouping of $\tilde{H}^{n}(X, \pi)$. Let $\mathscr{A}$ be the usual augmentation ideal in this groupring (i.e., the ideal generated by all elements of the form $g-1$ ). Then if it has category $\leqslant c$, we have, by (3.7), that there is a homomorphism of abelian groups $\mathbf{Z}\left(\tilde{H}^{n}(X, \pi)\right) / \mathscr{A}^{c+1} \rightarrow \tilde{H}^{m}(X, \rho)$.

From henceforth in this section, we will denote any of the maps in (4.1), (4.3) or (4.4) by $\beta$. Note that in each case $\beta(e)=0$.

Theorem (4.14), which follows after several lemmas, shows among other things, that if $Y$ is an associative $H$-space and if $f \in[X, Y]$ and has finite order then the map induced by $f$ in any homology or cohomology theory also has finite order provided either $X$ or $Y$ has finite category.

In what follows, when working with $H$-spaces we will generally write 1 instead of e. 
(4.5) Lemma. Suppose cat $X \leqslant r$ and let $Y$ be an associative $H$-space. Let $f, g$, $h \in[X, Y]$ with $f^{m}=1$ and $g^{n}=1$. Then

(i) $m^{r-a-b}(f-1)^{a+1}(h-1)^{b}=0=n^{r-a-b}(h-1)^{b}(g-1)^{a+1}$ in $\omega_{T}(X, Y)$ for $0 \leqslant a+b \leqslant r$.

(ii) If $m$ is odd, then $m^{r-a-b-1}(f-1)^{a+1}(h-1)^{b}=0$ in $\omega_{T}(X, Y)$, and if $n$ is odd, then $n^{r-a-b-1}(h-1)^{b}(g-1)^{a+1}=0$ in $\omega_{T}(X, Y)$, provided in both cases $0 \leqslant a+b<r-1$.

(Throughout, we use the convention that $(h-1)^{\circ}=1$ for any map $h$.)

Proof. By (3.7), since the multiplication induced by $Y$ is associative, we have $(f-1)^{i}(h-1)^{j}=0$ for $i+j \geqslant r+1$.

We have $1=f^{m}=((f-1)+1)^{m}=1+\sum_{k=1}^{m}\left(\begin{array}{c}m \\ k\end{array}\right)(f-1)^{k}$. Therefore

$$
\sum_{k=1}^{m}\left(\begin{array}{c}
m \\
k
\end{array}\right)(f-1)^{k}=0
$$

and hence

$$
\sum_{k=1}^{m}\left(\begin{array}{c}
m \\
k_{-}
\end{array}\right)(f-1)^{k+a}(h-1)^{b}=0
$$

where $a$ and $b$ are fixed with $0 \leqslant a+b \leqslant r$.

Now construct a sequence of equations beginning with (4.6) in which each equation is obtained from the previous one by multiplying on the left by $(f-1)$ and using the fact that $(f-1)^{i}(g-1)^{j}=0$ for $i+j \geqslant r+1$. We obtain

$$
\left.\begin{array}{c}
\sum_{k=1}^{m}\left(\begin{array}{c}
m \\
k
\end{array}\right)(f-1)^{k+a}(h-1)^{b}=0 \\
\sum_{k=1}^{m}\left(\begin{array}{c}
m \\
k
\end{array}\right)(f-1)^{k+a+1}(h-1)^{b}=0 \\
\vdots \\
m(f-1)^{r-h}(h-1)^{b}=0
\end{array}\right\}, \quad r-a-b \text { equations. }
$$

Consider the last two terms of (4.8), namely

$$
\begin{gathered}
m(f-1)^{r-b-1}(h-1)^{b}+\left(\begin{array}{c}
m \\
2
\end{array}\right)(f-1)^{r-b}(h-1)^{b}=0, \\
m(f-1)^{r-b}(h-1)^{b}=0 .
\end{gathered}
$$

Multiplying the first of these by $m$ and using the second we obtain

$$
m^{2}(f-1)^{r-b-1}(h-1)^{b}=0 .
$$

Continuing this procedure backwards through (4.7) we get

$$
m^{r-a-b}(f-1)^{a+1}(h-1)^{b}=0 .
$$

This proof is now easily modified to prove that $n^{r-a-b}(h-1)^{b}(g-1)^{a+1}=0$.

Now suppose that $m$ is odd and $0 \leqslant a+b<r-1$. Then $m$ divides $\left(\begin{array}{c}m \\ 2\end{array}\right)$ and hence (4.8) yields $m(f-1)^{r-b-1}(h-1)^{b}=0$. The previous procedure then gives $m^{r-a-b-1}(f-1)^{a+1}(h-1)^{b}=0$. Similarly we prove $n^{r-a-b-1}(h-1)^{b}(g-1)^{a+1}$ $=0$. 
Before discussing the situation when cat $Y<\infty$ we need the following lemma, which will also be useful in the sequel.

(4.9) Lemma. Suppose cat $Y=s$ and that $Y$ is an associative $H$-space. If $X$ is any space and $f \in[X, Y]$, then $(f-1)^{s+1}=0$ in $\omega_{T}(X, Y)$.

Proof. Apply (3.7) to the identity map in $[Y, Y]$ and then compose with $f$.

(4.10) LemmA. Suppose cat $Y \leqslant s$ and that $Y$ is an associative $H$-space. Let $f, g$, $h \in[X, Y]$ with $f^{m}=1$ and $g^{n}=1$. Then, for any $b$, we have

(i) $m^{s-a}(f-1)^{a+1}(h-1)^{b}=0=n^{s-a}(h-1)^{b}(g-1)^{a+1}$ in $\omega_{T}(X, Y)$ for $0 \leqslant$ $a \leqslant s$;

(ii) if $m$ is odd then $m^{s-a-1}(f-1)^{a+1}(h-1)^{b}=0$ in $\omega_{T}(X, Y)$ and if $n$ is odd then $n^{s-a-1}(h-1)^{b}(g-1)^{a+1}=0$ in $\omega_{T}(X, Y)$, provided in both cases that $0 \leqslant a$ $<s-1$.

Proof. The essential difference between this lemma and Lemma (4.5) is the fact that while, in the context of Lemma (4.5), $\prod_{i=1}^{r+1}\left(f_{i}-1\right)=0$ for any choice of $f_{i}$, in the current lemma all we can say is that $(f-1)^{s+1}=0$ for any particular function $f$.

The proof proceeds as in (4.5) up until (4.7), which becomes

$$
\left.\begin{array}{c}
\sum_{k=1}^{m}\left(\begin{array}{c}
m \\
k
\end{array}\right)(f-1)^{k+a}(h-1)^{b}=0 \\
\sum_{k=1}^{m}\left(\begin{array}{c}
m \\
k
\end{array}\right)(f-1)^{k+a+1}(h-1)^{b}=0 \\
\vdots \\
m(f-1)^{s-a}(h-1)^{b}=0
\end{array}\right\}, \quad s-a \text { equations. }
$$

The proof now continues as in (4.5).

We now extend this lemma in a way that will be useful when $[X, Y]$ is not abelian.

(4.11) Definition. Let $f \in[X, Y]$ and suppose $Y$ is an associative $H$-space. We will say that $f$ is $m$-generated if $f=h_{1} \cdots h_{a}$ with $h_{i}^{m}=1$.

(4.12) LEMMA. If, in the hypothesis of (4.5) and (4.10), we replace the conditions $f^{m}=1$ and $g^{n}=1$ by the conditions $f$ is $m$-generated and $g$ is $n$-generated, respectively, then the results hold for $a=0$.

Proof. We will only prove the first part of (i) of (4.5), the proof of the rest being nearly identical. Assume in general that $f=h_{1} \cdots h_{u}$ with $h_{i}^{m}=1$. The proof will be by induction on $u$. The case $u=1$ is immediate from (4.5). Suppose the result holds for $u=l$ with any choice of $h_{i}$. Let $f=h_{1} \cdot h_{2} \cdots h_{l+1}$ with $h_{i}^{m}=1$. By the induction hypothesis we have $m^{r-b}\left(h_{2} \cdots h_{l+1}-1\right)(h-1)^{b}=0$. Multiplying on the left by $h_{1}$ we get

$$
m^{r-b}\left(h_{1} h_{2} \cdots h_{l+1}-h_{1}\right)(h-1)^{b}=0
$$


or

$$
m^{r-b}\left((f-1)-\left(h_{1}-1\right)\right)(h-1)^{b}=0 .
$$

Hence our result.

We now deduce some consequences of (4.12). The functions given in the next definition will be used to provide estimates of the orders of certain elements.

(4.13) Definition. Fix $r, s \geqslant 0$, where $r$ and $s$ are allowed to be $\infty$. Under conditions given below, we define functions on the positive integers by

$$
\lambda_{d}(w)=w^{\min \{a \in\{r-d, s\} \mid a \geqslant 0\}} ; \gamma_{d}(w)=w^{\min \{a \in\{r-d-1, s-1\} \mid a>0\}}
$$

where $d$ can be either 0 or 1 . These functions are defined only when they make sense under the conventions that $\infty-k=\infty$ and $\min \{k, \infty\}=k$ for finite $k$. The minimum of the sets \{\} or $\{\infty\}$ is undefined. In particular, none of these four functions is defined when both $r$ and $s$ equal $\infty$.

(4.14) Theorem. Suppose cat $X \leqslant r$ and cat $Y \leqslant s$ where $r$ or $s$ is possibly infinite. Let $Y$ be an associative $H$-space. If $f, g, h \in[X, Y]$ with $f$ m-generated and $g$ $n$-generated, then, where the functions involved are defined, we have:

(1) $\lambda_{0}(m) \beta(f)=m^{\min (r, s)} \beta(f)=0$;

(2) if $m$ is odd, then $\gamma_{0}(m) \beta(f)=0$;

(3) $\lambda_{1}(m) \beta(h \cdot f)=\lambda_{1}(m) \beta(f \cdot h)=\lambda_{1}(m)(\beta(f)+\beta(h))$;

(4) if $m$ is odd, then $\gamma_{1}(m) \beta(h \cdot f)=\gamma_{1}(m) \beta(f \cdot h)=\gamma_{1}(m)(\beta(f)+\beta(h))$;

(5) if $L \subset[X, Y]$ is a closed subset of m-generated elements, then there are homomorphisms given by

(i) $f \rightarrow \lambda_{1}(m) \beta(f)$, and

(ii) $f \rightarrow \gamma_{1}(m) \beta(f)$ if $m$ is odd;

(6) g.c.d $\left(\lambda_{1}(m), \lambda_{1}(n)\right)(\beta(f \cdot g)-\beta(f)-\beta(g))=0$.

Proof. These are straightforward consequences of (4.12). We will prove (1) and (4) as examples.

(1) Taking $a=0=b$ in the first half of part (i) of (4.5) and (4.10) we obtain $m^{r}(f-1)=0$ for $r \geqslant 0$ and $m^{s}(f-1)=0$ for $s \geqslant 0$. Applying $\beta$ to each of these we get $m^{r} \beta(f)=0=m^{s} \beta(f)$. Therefore $m^{\min (r, s)} \beta(f)=\lambda_{0}(m) \beta(f)=0$.

(4) Take $a=0, b=1$ in part (ii) of (4.5) and (4.10) to get

$$
m^{r-2}(f-1)(h-1)=0=m^{r-2}(h-1)(f-1)
$$

for $r-2>0$ and

$$
m^{s-1}(f-1)(h-1)=0=m^{s-1}(h-1)(f-1)
$$

for $s-1>0$. Expanding and applying $\beta$ gives the result.

The following simple example suggests that at the price of involved calculations, one can obtain results similar to 4.14 in the case where $Y$ is not an associative $H$-space.

(4.15) Proposition. Suppose cat $X=2$ and that either $Y$ is an $H$-space (not necessarily associative) or that the (set) map $[Y, Y] \rightarrow[S Y, S Y]$ is a surjection. If $[X, Y]$ consists of two elements $e$ and $f$, then $K \cdot \beta(f)=0$ where $K=3$ or 4 . 
Proof. Assume first that $[Y, Y] \rightarrow[S Y, S Y]$ is surjective. Then consider $X \stackrel{f}{\rightarrow} Y \stackrel{i}{\rightarrow} \Omega S Y$, where $i$ is the inclusion. There is a map $P$ such that the following diagram commutes

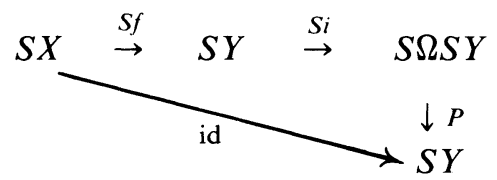

where id is the identity map.

Now $\Omega S Y$ is an associative $H$-space so we have the relation $(i \circ f-1)^{3}=0$ in $\omega_{T}(X, \Omega S Y)$.

$$
\therefore(i \circ f)^{3}-3(i \circ f)^{2}+3(i \circ f)-1=0, \text { and }
$$

$$
(i \circ f)^{4}-3(i \circ f)^{3}+3(i \circ f)^{2}-i \circ f=0 .
$$

Suspending these relations and then composing with $P$ we have, in $\omega_{T}(S X, S Y)$,

$$
\begin{aligned}
P \circ\left[S\left((i \circ f)^{3}\right)\right]-3 P \circ & {\left[S\left((i \circ f)^{2}\right)\right]+3 P \circ[S(i \circ f)]-e=0, } \\
P \circ\left[S\left((i \circ f)^{4}\right)\right]-3 P \circ & {\left[S\left((i \circ f)^{3}\right)\right] } \\
+ & 3 P \circ\left[S\left((i \circ f)^{2}\right)\right]-P \circ[S(i \circ f)]=0 .
\end{aligned}
$$

Since $[Y, Y] \rightarrow[S Y, S Y]$ is surjective, we have that $P \circ\left[S\left((i \circ f)^{n}\right)\right]$ is homotopic to either $S f$ or $e$. Suspending (4.17) and using (2.4) we see that we have two kinds of relations in $\left[S^{2} X, S^{2} Y\right]$, namely

$$
y-3 z+3 a=0 \text { and } x-3 y+3 z-a=0
$$

where $a=S^{2} f$ and $x, y$ and $z$ are equal to either 0 or $a$. We now proceed by cases, substituting values of $x, y$ and $z$ into (4.18), to obtain the following list:

$$
\begin{array}{llll}
y=a, z=a & \text { gives } & a=0 \\
y=a, z=0 & \text { gives } & 4 a=0 \\
y=0, z=0 & \text { gives } & 3 a=0 \\
x=a, y=0, z=a & \text { gives } & 3 a=0 \\
x=0, y=0, z=a & \text { gives } & 2 a=0
\end{array}
$$

In the case where $Y$ is an $H$-space we have a retraction $\Omega S Y \rightarrow Y$ and the proof proceeds along the same lines as before.

5. Spaces admitting two $H$-space structures. We now given an example of how to mix two $H$-space structures on a space $Y$ in order to produce relations in $\omega_{T}(X, Y)$.

As is well known (see [K]), $\tilde{K}_{\mathbf{R}}\left(\mathbf{R} P^{2}\right.$ ) is a ring generated by an element $\lambda$ with relations $4 \lambda=0$ and $\lambda^{2}=2 \lambda$. Suppose $\oplus$ and $\otimes$ are the two $H$-space structures on $B O$ induced by Whitney sum and tensor product of bundles. Then the multiplication, $a \cdot b$, of two elements $a, b \in \tilde{K}_{\mathbf{R}}\left(\mathbf{R} P^{2}\right)$ is determined by $a \cdot b=a \otimes b-a$ - $b$. Using this, one can easily work backwards to construct the multiplication table 
for $\left[\mathbf{R} P^{2}, B O\right]$ with respect to the $\otimes$ structure on $B O$. The tables for the multiplications given by $\oplus$ and $\otimes$, given below, are written multiplicatively in order to conform with our previous notation.

\begin{tabular}{c|c|c|c|c|}
$\oplus$ & 1 & $\lambda$ & $\alpha$ & $\gamma$ \\
\hline 1 & 1 & $\lambda$ & $\alpha$ & $\gamma$ \\
\hline$\lambda$ & $\lambda$ & $\alpha$ & $\gamma$ & 1 \\
\hline$\alpha$ & $\alpha$ & $\gamma$ & 1 & $\lambda$ \\
\hline$\gamma$ & $\gamma$ & 1 & $\lambda$ & $\alpha$ \\
\hline
\end{tabular}

\begin{tabular}{c|c|c|c|c|}
$\otimes$ & 1 & $\lambda$ & $\alpha$ & $\gamma$ \\
\hline 1 & 1 & $\lambda$ & $\alpha$ & $\gamma$ \\
\hline$\lambda$ & $\lambda$ & 1 & $\gamma$ & $\alpha$ \\
\hline$\alpha$ & $\alpha$ & $\gamma$ & 1 & $\lambda$ \\
\hline$\gamma$ & $\gamma$ & $\alpha$ & $\lambda$ & 1 \\
\hline
\end{tabular}

We will use $\oplus$ and $\otimes$ to denote the corresponding multiplications induced in $\omega_{T}\left(\mathbf{R} P^{2}, B O\right)$. Since $\mathbf{R} P^{2}$, has category 2 , the following relations must hold in the multiring $\omega_{T}\left(\mathbf{R} P^{2}, B O\right)$.

$$
\begin{aligned}
0 & =(\alpha-1) \otimes((\lambda-1) \oplus(\gamma-1)) \\
& =(\alpha-1) \otimes(\lambda \oplus \gamma-\lambda-\gamma+1) \\
& =\alpha \otimes(\lambda \oplus \gamma)-\alpha \otimes \lambda-\alpha \otimes \gamma+\alpha-\lambda \oplus \gamma+\lambda+\gamma-1 \\
& =\alpha-\gamma-\lambda+\alpha-1+\lambda+\gamma-1 \\
& =2 \alpha-2 .
\end{aligned}
$$

Therefore $2 \alpha=2$.

Applying $\otimes \gamma$ to both sides we obtain $2 \lambda=2 \gamma$.

From $(\alpha-1) \otimes(\gamma-1) \otimes(\gamma-1)=0$ we easily obtain $4 \gamma=4$.

After applying $\beta$ we obtain the following relations: $2 \beta(\alpha)=0,2 \beta(\lambda)=2 \beta(\gamma)$ and $4 \beta(\gamma)=0$. It is not at all clear whether or not this is a complete set of relations. This is a major problem in general, since the number of possible relations grows extremely fast as the category increases.

6. The Hopf construction. From a map $f: X \times Y \rightarrow Z$, the Hopf construction produces a map $T f: X^{*} Y \rightarrow S Z$ (see [W]). The following is routine:

(6.1) Proposition. The map $\omega_{T}(X \times Y, Z) \rightarrow \omega_{T}\left(X^{*} Y, S Z\right)$, given by $f \rightarrow T f$ is a well defined homomorphism of abelian groups.

(6.2) Proposition. Let $X$ and $Y$ be any spaces and suppose $\bar{a}: X \rightarrow S^{1}, \bar{b}: Y \rightarrow S^{1}$. Define $a: X \times Y \stackrel{\text { proj }}{\rightarrow} X \stackrel{\bar{a}}{\rightarrow} S^{1}$ and $b: X \times Y \stackrel{\text { proj }}{\rightarrow} Y \stackrel{\bar{b}}{\rightarrow} S^{1}$. Then $2 S^{2} T(a \cdot b)=0$ in $\left[S^{2}\left(X^{*} Y\right), S^{3} Z\right]$. If (2.5) applies, then $2 S T(a \cdot b)=0$ in $\left[S\left(X^{*} Y\right), S^{2} Z\right]$. 
Proof. By (4.9) we have $(h-1)^{2}=0$ in $\omega_{T}\left(X \times Y, S^{1}\right)$, for any $h: X \times Y \rightarrow S^{1}$. Therefore $h^{2}-2 h+1=0$ and hence $h^{-1}=-h+2$. Now

$$
\begin{aligned}
a b & =\left(b^{-1} a^{-1}\right)^{-1}=-b^{-1} a^{-1}+2 \\
& =-(-b+2)(-a+2)+2=-b a+2 a+2 b-2 .
\end{aligned}
$$

However, $a b=b a$ and therefore $2 a b=2 a+2 b-2$ in $\omega_{T}\left(X \times Y, S^{1}\right)$. Applying the Hopf construction, we obtain $2 T(a \cdot b)=2 T(a)+2 T(b)-2$ in $\omega_{T}(X \times Y, S Z)$ by (6.1). Since $a$ and $b$ factor through projections, by standard properties of the Hopf construction $T(a) \sim T(b) \sim 1$ and hence $2 T(a \cdot b)=2$. The result now follows from (2.4).

Recall that $\eta \in \pi_{3}\left(S^{2}\right)$ can be defined as $T f$ where $f: S^{2} \times S^{1} \rightarrow S^{1}$ is given by $(x, y) \rightarrow x y^{-1}$. (6.2) shows that $2 S^{2} \eta=0$, as is well known.

Let $x, y: S^{n} \times S^{n} \rightarrow S^{n}$ be the projections onto the first and second factors, respectively. For $n=1,3,7$ the element in $\pi_{2 n+1}\left(S^{n+1}\right)$ which is the Hopf construction of an arbitrary product of powers of $x$ and $y$ can be determined using the E.H.P. sequence and cohomology. Our techniques give a simple method for computing the element in $\pi_{2 n+2}\left(S^{n+2}\right)$ obtained after suspending once. As an example of this type of calculation consider $x(y x): S^{7} \times S^{7} \rightarrow S^{7}$.

We have $(x-1) \cdot((y-1) \cdot(x-1))=0$ in $\omega_{T}\left(S^{7} \times S^{7}, S^{7}\right)$ since $S^{7} \times S^{7}$ has category 2. Recall from the proof of (6.2) that $y x=-x y+2 x+2 y-2$. Therefore

$$
\begin{aligned}
x(y x) & =x y+x^{2}+y x-x-y-x+1 \\
& =x y+2 x-1+(-x y+2 x+2 y-2)-x-y-x+1 \\
& =2 x+y-2 .
\end{aligned}
$$

Applying the Hopf construction $T$ we get

$$
T(x(y x))=2 T(x)+T(y)-2 \text { in } \omega_{T}\left(S^{15}, S^{8}\right)
$$

by (6.1). Since $x$ and $y$ factor through projections, by standard properties of the Hopf construction $T(x) \sim T(y) \sim 1$ and hence $T(x(y x))=1$. Applying (2.4), we have $S^{2} T(x(y x))=0$ in $\pi_{17}\left(S^{10}\right)$.

7. Cobordism. Suppose $M$ is a smooth manifold with basepoint. (For this theory it would be possibly better to use the basepoint free version of the results in $\$ 3$ which are easily developed.) Let $h: M \rightarrow \mathbf{R}^{n+k}$ be an embedding with normal bundle classified by $M \stackrel{{ }^{\prime \prime h}}{\rightarrow} G_{k, n+k} \hookrightarrow B O(k)$ and suppose that $\tilde{v}_{n}$ is a trivialization of $v_{n}$, i.e., the following diagram commutes:

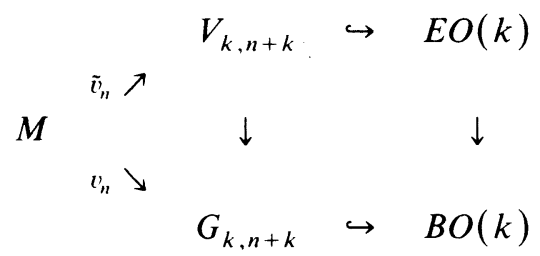

Let $f \in[M, O(k)]$. By multiplying in the fiber, $O(k)$, of $V_{k, n+k} \rightarrow G_{k, n+k}$ we can modify $\tilde{v}_{n}$ to obtain $f \cdot \tilde{v}_{n}$. If we think of $\left(h, \tilde{v}_{n}\right)$ as fixed, then it turns out that there is a homomorphism of abelian groups $\sigma_{k}: \omega_{T}(M, O(k)) \rightarrow \pi_{*}^{s}\left(S^{0}\right)$ given by $f \rightarrow$ $P T\left(h, f \cdot \tilde{v}_{n}\right)$ where PT denotes the Pontrjagin-Thom construction. Since $\sigma_{k}$ extends 
to $\sigma: \omega_{T}(M, O) \rightarrow \pi_{*}\left(S^{0}\right)$, we have a map that relates the group $[M, O]$ and the category of $M$ in a nontrivial way to the element in the stable homotopy groups of spheres produced by the Pontrjagin-Thom construction. We can now easily produce results similar to Theorem (4.14), the only difference being that $\sigma(1) \neq 0$ in general. For instance if cat $M \leqslant r$ and $f \in[M, O]$ with $f^{m}=1$ then from the relation $m^{r}(f-1)=0$ in $\omega_{T}(M, O)$, we obtain $m^{r} P T\left(h, f \cdot \tilde{v}_{n}\right)=m^{r} P T\left(h, \tilde{v}_{n}\right)$, after applying $\sigma$.

These aspects of this theory, especially the connections with products in cobordism and stable homotopy are currently under investigation.

The author wishes to acknowledge the support and suggestions of Eddy Campbell, Peter Hoffman, Richard Kane, Paul Selick and Victor Snaith.

\section{BIBLIOGRAPHY}

[K] Max Karoubi, K-theory. An introduction, Springer-Verlag, Berlin-New York, 1978.

[R] William J. Ralph, An extension of singular homology to Banach algebras, Pacific J. Math. (to appear).

[St] James Stasheff, $H$-spaces from a homotopy point of view, Lecture Notes in Math., Vol. 161 , Springer-Verlag, Berlin and New York, 1970.

[Sw] Robert M. Switzer, Algebraic topologv-homotopy and homologv, Springer-Verlag, New York and Heidelberg, 1975.

[W] George W. Whitehead, Elements of homotopy theory, Springer-Verlag, New York and Heidelberg, 1978.

Department of Mathematics, The University of Western Ontario, London, Ontario, Canada, N6A $5 B 7$

Current address: Department of Mathematics, Brock University, St. Catharines, Ontario, Canada L2S $3 \mathrm{~A} 1$ 